\section{Briginal surtitleg.}

\section{STOKES-ADAMS SYNDROME.*}

A REPORT OF TWO CASES WITH A SHORT RÉSUMÉ OF THE LITERATURE.

BY T. J. FOLEY, M.D., WORCESTER, MAs8. Visiting Physician St. Vincent's Hospital, Worcester City Hospital Out-Patient Department, Worcester Isolation Hospital.

I HAvE been fortunate enough to meet with two striking examples of this peculiar group of symptoms and I take this opportunity to report them to you. I also include in this article a short résumé of the literature with the purpose of calling your attention to the variety of pathological conditions that may be found as a probable cause.

The disease is characterized by a vertigo, syncope, loss of consciousness and a slow pulse a pseudoapoplexy. Riegel, ${ }^{1}$ taking 60 as the normal pulse, found a total of 1,041 in 7,567 examinations where the pulse was below that, but of these, only 47 had disease of the organs of circulation. We have all perhaps run across people with permanent slow pulse, in whom it is apparently physiological or, as Mayo ${ }^{2}$ says guardedly, is " compatible with either ordinary length of days, or, at all events, with the continuance of life for an indefinite period."

I knew a young man, always in fine health, whose pulse, as a rule, was 50 that rose to 60 at the beginning of an acute appendicitis and the sudden appearance of a pulse of 70 on the second day I felt was of grave significance, although his general condition had not apparently become any worse. He was at once transferred to the hospital, and an immediate operation disclosed a gangrenous appendix and free pus. He made a very slow recovery. R. T. Edes ${ }^{3}$ mentions the fact that Napoleon's normal pulse was about 40 and comments on the strange attacks of depression and apathy that assailed him on more than one occasion. A possible connection between his slow pulse and epileptiform seizures is also suggested. Vigoroux ${ }^{4}$ mentions a laborer whose pulse and heart beats were 20 , who was capable of great exertion and was never sick. There are a number of such cases recorded but with these I will not concern myself in this paper. We have to do more with the pathological forms in which either clinically or anatomically have been demonstrated cardiac lesions, with or without atheroma of the aorta and coronary arteries, or, in rare instances, changes in the nervous system. We may say then that the Stokes-Adams syndrome may be either of nervous or cardiac origin.

One of the cases that came under my observation appeared at the Out-Patient Department of the City Hospital, Dec. 8, 1904. He was first seen by the house officer, who obtained this history from him: He was fifty-seven years of age and a wire worker by occupation. His wife became insane one year before and since then he has become very nervous. Previous to that time his personal history as far as sickness was concerned was very good. His present illness began about six months before, with attacks of vertigo, 1905 .

* Read before the Worcester District Medical Society, April 12 followed by pain in the chest and headache. The attacks were more noticeable on rising in the morning or when bending over. He thinks he sometimes became unconscious in these attacks. The pupils react well, tongue tremulous - protrudes in the median line, slightly coated. Pulse was 38 , full and regular. Heart displaced toward the left. I saw him the following day. He was tall and poorly nourished. I found the cardiac area to be enlarged, the left heart one finger's breadth outside the nipple line; the right heart to the middle of the sternum. No thrill could be detected. There was a rather sharp first sound at the apex, followed by an exquisitely soft systolic murmur, the latter also being heard over the preoordia. Both second sounds were weak but pure. The heart beat was perfectly synchronous with the pulse. When the patient assumed the horizontal position his pulse was 32 , and on exercise went up to 44 . His lungs showed signs of a moderate amount of emphysema. Liver and spleen were not enlarged. There was no edema. My anatomical diagnosis was myocardial degeneration resulting from sclerosis or calcification of the coronary arteries. There was probably also some degree of atheroma of the aorta. It is impossible in these cases to exclude the possibility of changes in the ganglia of the heart, also a result of the coronary changes, and further mention of such changes will be made later on. The systolic murmur I considered to be myocardial in origin. The left heart only was enlarged and there was no accentuation of the second pulmonic tone. I saw very little of the patient afterwards but I mention his case as furnishing the most common example of the disease. He was transferred to the house medical side for observation, and through the kindness of Dr. Thomas Howell I am able to give you some idea of the further history of this patient. He was kept in bed for a time and the house officer gave him for experimental and diagnostic purposes a short course of treatment with atropine, but it had no appreciable effect on his pulse. Rest in bed however seemed to relieve his vertigo, and he made no report of such attacks until Jan. 6, three weeks after his admission into the hospital. He had one slight feeling of dizziness that morning but it was not noticed by any of the hospital attendants. From this time on he was up and about the ward, having occasional attacks of vertigo but no periods of unconsciousness until Jan. 30, he was sitting in a wheel chair and without any complaint of pain of any description suddenly collapsed. The house officer was close at hand, the man was taken from the chair, laid on the floor, atropine and brandy injected subcutaneously and artificial respiration practiced. The patient gasped a few times and was dead. His highest recorded pulse was 52 , and this was taken the day he went to the hospital, and it slowly declined until for the last few weeks before his death it varied from 20 to 30 . He was never seen in any of his dizzy attacks and it is impossible to say whether he had at any time a much slower pulse. His urine was always cloudy, acid, of a high specific gravity and contained neither sugar, albumin, or casts.

It is interesting here to notice the effect of the atropine and the use it may have for diagnostic purposes. According to. Dehio ${ }^{5}$ atropine is known to paralyze the inhibitory functions of the vagus from one end to the other. If atropine is given in a case where an intracranial lesion irritates the vagus the pulse quickens, while in cases of permanent slow pulse there is a slight result if any at all.

My other patient was a young man nineteen years of age, a machinist by occupation. His family history is of little interest. His father died of an abdominal tumor at fifty-seven years of age, a sister died of what was called "salt rheum," and a brother died of pul. 
monary tuberculosis. His mother and two brothers are alive and well. $\mathrm{He}$ is supposed to have had brain fever when eight years of age. He was a welldeveloped and well-nourished young man and had been in excellent health. On Jan. 13, 1901, he was taken with "sore throat" and when I saw him on the following evening there was considerable membrane on both tonsils. He complained of much pain and the cervical glands were enlarged. Temperature 103.2; pulse 118; it was a nasty-looking throat and, without waiting for a report from the culture, I at once gave 3,000 units of antitoxin; the following morning there was some improvement, temperature 101 ; pulse 100 ; culture was reported negative. Jan. 16, the temperature and pulse were normal and the second culture was reported negative. A third culture was taken Jan. 17, and was reported positive. By this time the membrane had entirely disappeared from the throat and the patient was up and about the house. I found it impossible to control him and made no attempt to do so after the fourth and final culture was taken. From the time shortly after iny last visit until April 23, about three months, he had felt pretty well, although at no time as well as before his sickness. He worked steadily however. On the evening of this last date he made some complaint of pain in the stomach; when he got up on the following morning he felt well, but while putting on his shoes he suddenly pitched forward on the floor; he did not lose consciousness and soon revived. At this time he experienced severe pain in his head and chest. This was soon followed by a sinking feeling and he thought he was going to die. When I saw him, about an hour after his first attack, he was very pale, was sweating profusely and felt nauseated. He thought he would feel better if he could vomit and tried to induce vomiting by putting his finger back in his throat. He was nervous and uncomfortable, and, against my protest, got up out of bed and sat on a vessel; in a few moments he suddenly pitched forward on the floor. His body assumed the position of opisthotonos but the movement remained tonic in character, the respiration ceased and the face became ghastly pale at first, gradually changing to a cyanotic hue as the rigidity increased in intensity. $\mathrm{He}$ looked as though he was about to die. Finally, after an interval of twenty to thirty seconds, the muscles began to relax, respirations began, at first spasmodically, then a few deep breaths and consciousness returned. After being placed in bed his pulse was 16, the heart was found to be enlarged, the left heart reaching one finger's breadth outside the nipple line and the right heart to the right edge of the sternum. At the apex the first sound was sharply accentuated, valvular in quality and split; the other cardiac sounds were feeble but pure, there was no murmur, but occasionally a sound not synchronous with the pulse beat was heard. Unfortunately no examination was made of the veins of the neck and I could not say whether these intermediate beats were auricular or not. Edes mentions Chauveau and also Vaquez and Bureau as authorities, who say that the small intermediate beats which could be seen in the cardiogram and synchronously with this in the jugular pulsations were referable to the auricular contractions which were not communicated to the ventricles, and this condition of things was compared to what is found when the vagus of the horse is weakly stimulated - that is the inhibition of the ventricular contractions, while the auricular are but little affected. My patient had seven attacks during the day but finally, under the influence of morphia and strychnia, he gradually improved until after his last attack, when his pulse was 24 , there was a gradual rise to 40 the next morning. He was kept under observation for one week; the pulse was never higher than 42 , always irregular in rhythm and quality. Temperature was always slightly subnormal. Urine, acid, specific gravity 1,018 , contained no sugar, albumin, or casts. One month afterwards I met him on the street and he was hurrying along at a rapid rate. He had another attack June 19, 1903 , in which he did not lose consciousness and when I saw him his pulse varied from 30 to 36 . He was fairly comfortable, however, did not suffer any pain and after a week's rest in bed was allowed to get up again. The cardiac area was smaller than it was two years before. I have lately examined this patient. His heart area is normal, pulse varies between 80 to 90 , while the heart sounds are perfectly normal.

F. W. White and H. A. Smith ${ }^{6}$ have made a study of heart changes in nearly one thousand cases of diphtheria and tell us that murmurs, rapid and irregular pulse are common. The majority of the murmurs, which were usually systolic and were heard best at the apex, were accompanied by an accentuated second pulmonic tone and irregular heart action that outlasted the fever. They reported 3 cases of bradycardia occurring usually at the end of the second week or the beginning of the third week. The pulse ranged at that time from 20 to 30 and in all cases a fatal ending resulted. Not any of the three patients showed any typical symptoms of StokesAdams disease except the bradycardia. In the discussion that followed Dr. McCollom said he considered the majority of the heart symptoms to be due to a change in the pneumogastric nerve. I have never to my knowledge seen a case of diphtheria at an autopsy where endocardial changes have taken place. Gibson ${ }^{7}$ refers to the frequency of endocarditis during the acute infectious diseases. Out of 670 cases in the Leipzig Clinic, Romberg ${ }^{8}$ says $58.5 \%$ were rheumatic, $12.3 \%$ arteriosclerotic, $3 \%$ were due to chorea, smallpox and gonorrhea, while less than $.3 \%$ were due to scarlet fever, diphtheria and septicemia. Baginsky $^{9}$ in his fine work on diphtheria says the disease can cause almost any anatomical lesion of the heart. Most of the changes however are myocardial, consisting of a fatty degeneration of the muscle fibers, a great increase in round cells in the interstitial tissue and well marked changes in the muscle nuclei. Romberg ${ }^{10}$ also gives a prominent place to diphtheria as a cause of myocarditis. He says the latter disease is almost always brought about by infectious diseases. He thinks, however, that coronary sclerosis as a cause of the chronic inflammation of the heart muscle has not been given the attention it deserves. These heart degenerations are in general more difficult to diagnose clinically from endocardial disease. Many cases of diphtheria myocarditis are accompanied by a systolic murmur with an accentuated second pulmonic tone, but Baginsky explains them as being an expression of an inexactly placed mitral valve, or damage to the papillary muscle brings about an insufficiency of the mitral valve. The further history of the case is usually enough to differentiate the two conditions, but it is a difficult matter to explain why a myocarditis is accompanied in one case by a rapid pulse and in another 
by a slow pulse. Baginsky divides the heart paralysis very conveniently into the early paralysis, those cases in which the paralysis occurs while the local symptoms are present, and the late paralysis, where the heart as well as the other organs gives way to the steady progressive effects of the diphtheria poison. In the former class he makes no mention of a slow pulse but in the latter he makes note of a number of cases where the pulse was recorded 36 to 40 . In the great majority of cases he thinks the myocarditis present is enough to account for the heart paralysis, but it is not true in all cases, since he has been able to convince himself by frequent examinations that many of the early deaths occur without any noticeable change in the heart muscle; the more protracted the case, the greater the anatomical lesions, and it is to the muscle degenerations that those cases belong which resist death most successfully. My own case was given good-sized doses of morphine and atropine without any noticeable effect on the pulse, and if it is true that the atropine test has diagnostic significance, I feel that I had to do with the myocarditis and that there was little of any change in the vagus. Adams' original case in 1827 was sixty-eight y ears old, while Stokes' ${ }^{\prime 1}$ two cases, that led to his famous monograph on slow pulse, were fifty and sixty-eight. His description of these cases makes interesting and instructive reading to-day. In these early cases fatty changes in the heart were prominent, but it was Huchard ${ }^{12}$ who brought the symptoms to notice again in 1893 , who was strongly of the opinion that the nervous system took some part in the process, arterio-sclerotic in nature, influencing the brain and especially the medulla. Hans Luce $^{13}$ of Hamburg has in the Archiv für Klinische Medicin a very thorough clinical and anatomical description of a case together with an interesting résumé of some of the literature of the disease. He thinks the occurrence in heart cases (mostly of old individuals) of bradycardia breathing anomalies and loss of consciousness confines the disease within too narrow limits, that a number of variations are necessary to fill out a complete picture. Bradycardia is the most important symptom and about this symptom is grouped others. According to his perusal of literature, he considers the following the most important: Bradycardia with simple weakness or fainting -- bradycardia with apnea or with Cheyne-Stokes breathing or with irregularity of breathing - bradycardia with dizziness or headache - bradycardia with angina pectoris or cardiac asthma - bradycardia with apoplectic attacks or temporary hemiplegia (occurring, as one case shows, without anatomical findings) bradycardia with apoplectic attacks, epileptic seizures, with mydriasis, loss of pupil reflex and breathing anomalies. Huchard's "formes frustus" is a bradycardia with simple weakness or with attacks of precordial distress with irregularity of the heart, or attacks of extreme paleness which are combined with dizziness, with alterations of sensibility, and a high degree of general weakness. Most cases are found in old individuals, as they were in the original cases of Stokes and Adams, the anatomical lesions being sclerosis, calcification, atheroma of the aorta and of the coronary arteries together. Lipomatosis of the heart muscle is frequent. But a number of cases proves that it may occur at all ages, with different heart lesions. Von Schuster's ${ }^{14}$ little patient, a girl four years, was born healthy and remained so until an attack of rheumatic fever, when she later developed symptoms of myocarditis, and after passing through some severe attacks of Stokes-Adams disease, lasting for many months, finally recovered. Hoffman's patient was a girl twenty-three years of age, who had scarlet fever, diphtheria, ty phoid fever and recurring attacks of chlorosis in her history; she also recovered. Hoffman controlled his pulse finding by the $x$-ray. The course of the disease is very various - it may be extremely fulminating, especially in old people, the attacks coming suddenly, death taking place after a few hours or days; other patients may experience a number of attacks, the end coming as the attacks increase in rapidity; there are other cases, such as Hoffman's and Von Schuster's, where the attacks come suddenly, persist for weeks and months and disappear as suddenly as they came leaving no trace of their former presence. Attacks may come on after longer or shorter periods, sudden death finally taking place in a recurring attack. It is said that during an attack the pulse undergoes many changes, the bradycardia may become exaggerated, or the pulse quality becomes small, irregular, unequal or interrupted for the fraction of a minute or even for several minutes. Attention has been called to the disorders of the nervous system as cause of this disease and in looking over the literature a striking example of this form of the disease was found. Krause has also described a case with all the appearance of Stokes-Adams syndrome that occurred during an attack of uremia pathology. Adams' case was a lipomatosis cordis with atrophy and fatty degeneration of the heart muscle. Korcziniski ${ }^{16}$ observed a woman thirty-eight years of age with mitral stenosis and aortic insufficiency who suddenly suffered a stenocardial attack, pulse 24 , became unconscious, with mydriasis and spasms, died; autopsy - embolus of one coronary artery. Hammer ${ }^{17}$ saw a case in 1878 that collapsed; thirty hours before death had a pulse of 8; autopsy $-a$ vegetation of the aortic valve obstructed the left coronary artery causing a thrombus formation. Samuelson,,$^{18}$ treated a man forty-seven years of age who in his thirtieth year, during an inunction cure, experienced three times attacks which consisted in sudden loss of consciousness, slight delirium, dyspnea and clonic contractions of the face which lasted one half hour. During the following year this patient suffered frequent attacks of angina pectoris and cardiac asthma. During all this time there was nothing striking about his pulse; on the day of his death, after a hearty meal, he 
experienced a severe stenocardial attack,pulse 35,- became unconscious and died. Autopsy - an enormous sclerosis of the coronary artery with a high degree of narrowing of its lumen, directly after it left the aorta, with endarteritis obliterans in the further course of the artery. There were also isolated spots of scar formation and interstitial lipomatosis. Van Frev's ${ }^{10}$ patient, sixty years of age, had a number of attacks in four years. Autopsy - slight dilatation of the left ventricle, lipomatosis cordis with fatty degeneration and narrowing of the coronary arteries and considerable atheromatous deposits; hydrocephalus internus and externus. In all these cases no microscopical examination of the nervous system was made. The changes in the nervous system causing this disease are usually located in the pons, medulla, upper cervical cord and peripheral vagus branches. Hoberton found at the autopsy of a man sixtyfour years of age, in whom, three years after a fall from a horse, Stokes-Adams syndrome had set in, a narrowing of the occipital foramen, a thickening of the vagus and swelling of the upper cervical ganglion. Lepine found in a case a compression of the pons medulla from a jutting forward of the apophysis. Boyd described a case with syphilitic new formation on the floor of the fourth ventricle with 14 to 24 pulse which occasionally ceased for fifteen seconds; in none of these cases was an examination of the medulla made. A classical observation of the neurogenous Stokes-Adams symptom complex both from a clinical as well as an anatomical standpoint was furnished by Neuberger and Edinger ${ }^{20}$. A man, forty-six years of age, was being treated for chronic constipation and neurasthenia. During and after defecation he began to have fainting spells; pulse small, seldom over 60 , became weaker during the attacks; heart had no signs of organic lesions. Nine days before death he had a severe attack. Mydriasis, loss of pupil reflex, head and eyes turned to the left, pulse good and strong, 18. Pulse ceased at times during the attack, gradually returned and in the evening was 60 to 70 . Attacks almost continuous a few days before death; pulse 12 to 16, with stimulants, 30 to 40 . Autopsy - heart perfectly normal; almost total loss of right half of cerebellum. The most important finding was in the cervical cord. The capillaries of the gray substance about the central canal were filled with blood; it was further found in other sections in the neighborhood of the pyramidal crossing in the posterior horn between the base of it and the substantia gelatinosi Rolandii a large varix passing into a strongly filled venous plexus; the varix was the size of a hempseed. There was beginning hemorrhagic infarction of the tissue between. Its position was such that pressure was exerted on the upper bundle of the nervous accessorious Willisii and thence to the neighboring nuclei. It is easy to imagine how straining at stool was enough to bring about irritation of the inhibitory fibers of the vagus. This case proves, first, that in man the inhibitory fibers of the vagus arise from the nervous accessorious, and, second, the Stokes-Adams symptom complex can be brought about solely by causes centrally operating in the medulla. Zurhelle ${ }^{21}$ speaks of a case where swollen bronchial and mediastinal glands were the exciting factors. Recovery took place with hoarseness and paralysis of the recurrens. Luce's ${ }^{22}$ patient was a man fifty years of age; in his twenty-second year he had a hard cancer and in his twentyfifth year a skin eruption which disappeared under treatment. On examination he was found to be strikingly pale, pulse 28, cardiac area normal, loud systolic murmur, heard best over base and right of sternum, second sounds difficult to hear, slight liver enlargement, urine free from sugar or albumin, otherwise apparently normal. After admission to the hospital he had several attacks of unconsciousness, pulse slower, sometimes ceasing completely, irregular and of equal intensity; attacks lasted from one to two minutes. Several days after admission to the hospital, after a day of freedom from the attacks, he suddenly had repeated seizures and died. $\mathrm{Au}-$ topsy showed adhesions between right lung and costal pleura. But the interesting conditions were in the heart. The subparicardial fat in the entire anterior surface of the right ventricle strongly developed, in the posterior surface of the left ventricle only in the course of the vessels. Heart was a trifle enlarged. Below and to the right of the exit of the pulmonary artery on the anterior surface of the right ventricle was a tumor about the size of a walnut and lying below the epicard. A second smaller tumor was seen close by in the space between the origin of the aorta and the pulmonary arteries, in fact at the base of these vessels. Above and closely intimate with this tumor was another large tumor having its origin in the anterior and right wall of the aorta where the right auricle is accustomed to come in contact with the artery. On opening the heart a tumor was found projecting into both ventricles and finding its origin in the upper third of the ventricular septum. The tumor springing from the aorta was an aneurism. All the valves were found to be healthy. The beginning portion of both coronary arteries were completely free and no intima changes that might cause narrowing or deformity could be demonstrated. There were no enlargements or metastases in glands. Microscopical examination showed the heart tumors to be round-celled sarcomata. The kidneys showed a well-marked venous stasis and a fresh parenchymatous nephritis. We have here in the heart, produced a real heart stenosis, a pathological-anatomical rarity. It is curious that in spite of its origin and its intimate association with the blood stream there were no anastomoses outside the heart. There were others beside these mentioned in the heart but mostly of microseopical size. Very striking was the severe partial parenchymatous degeneration of both vagus nerves going from the upper thoracic aperture to its entrance into the medulla. The intramedullary motor and sensory vagus roots were 
intact. The same was true of the extra and intramedullary root fibers of the nervus accessorius Willisii. The vagus accessorius nuclei showed no histological change. No changes either could be made out in the peripheral vagoaccessory fibers. The condition here is the so-called retrograde or secondary degeneration brought about by the tumor in the ventricular septum. Luce tells us that this case has furnished us with important anatomical information. Up to this time the intracardial course of the vagus accessory nerves in the heart of man was unknown. Embryological studies showed us they ended in the auricles in numerous ganglia and by further development spread to the auricular septum in the neighborhood of the foramen ovale. They, apparently, had no connection with the ventricles. Luce insists that the peculiar degeneration of the vagus fibers proves that the course of the greater portion of the intracardial vagus fibers is through the ventricular septum. Ganglion cells are wanting in the ventricles and according to his theory the vagus fibers influence the heart muscle fibers directly and not through the medium of ganglionic cells. This is a point of academic interest since physiological experiment has not succeeded in clearing it up. Neither can the vagus be the trophic nerve of the heart since this heart, in spite of the long history of the case (two years) was trophically normal and examination showed not the slightest sign of fatty degeneration. The physical examination of this patient gave a heart that was not enlarged, the impulse at the apex was weak and there was no thrill to be felt. Over all the heart area was heard a loud systolic murmur, heard best at the base or to the right side of the sternum. Aortic and pulmonary stenosis were excluded by the absence of hypertrophy or dilatation of left or right ventricles, absence of stasis, and the good full pulse. It is certainly remarkable in this case how regulation and compensation of the circulation were carried on. Luce suggests that the bradycardia is a toxic myasthenia of the heart muscle caused by the sarcoma and toxic products resulting from it. He cites cases of sarcoma of different organs that were accompanied by Stokes-Adams symptoms during life. As far as the nervous symptoms of Stokes-Adams are concerned individual disposition of the nervous system is of importance (Luce). Individual tolerance of different organs varies within wide limits. Japanese physicians report that the coolie because of his great exertions has a breathing center that is difficult to influence. They endure pneumonia, even with extensive involvement of the lung, with comparatively little dyspnea. The same is true of divers.

Naunyn ${ }^{23}$ was able in three patients above fifty years of age by digital compression of the carotid at the throat to produce bradycardia, loss of consciousness, interruption of breathing and sometimes severe twitching of the extremities. This he was unable to do with younger patients. The diversity of the nervous symp- toms of Stokes-Adams symptom complex may be considered as an expression of an individual nervous disposition which can be hereditary, or acquired through the presence of pathological conditions (such as arterio-sclerosis and chronic blood-vessel disturbances of any kind). This latter may explain why in one case convulsions are present, why they fail in another.

A case of acute dilatation due to exertion was reported by Strübing ${ }^{24}$ of a boy who, after a hard run, fainted and afterwards had attacks of loss of consciousness and convulsions. He later had attacks with vertigo and headache. Pulse 16 to 18. He died in a paroxysm. No autopsy. A case was reported as caused by heavy lifting. Mental and emotional strain have been mentioned as causal factors but I have seen no case reported. The prognosis has been touched upon. It rests entirely on the study of the individual case - the condition of the heart, the severity of the symptoms and the condition of the pulse during the paroxysms would give the most important results (Edes).

The treatment consists in rest; diet, tonics and regulation of exercise are sometimes important. Some of the older patients may live for years but they must guard themselves against bodily or mental worry. This is especially true of the coronary cases. John Hunter, the great pathologist, was a victim of coronary sclerosis and suffered from stenocardial attacks. His statement "that his life lay in the hands of any rascal who chose to tease or annoy him" shows how precarious is such a patient's hold on life and how finely adjusted is the balance between the inhibitory and the augmentor fibers. In the developed paroxysms, the cardiac stimulants, like ammonia and the nitrates, are useful. Atropin may be given subcutaneously. The recumbent posture favors the return of blood to the brain. One patient of ten prevented an attack by getting on his hands and knees and allowing the head to hang down. Cases are known where rest and good feeding caused disappearance of attacks but without any change of the pulse.

REFERENCES.

1 Riegel: Zeitschrift für Klin. Medicine, 1890, p. 22

2 Riegel: Zeitschrift für Kin. Medicine, 1890, p. 22.

2 Mayo: London Medical Gazette U. S., Vol. xxii, p. 232. 1901, Vol. xvi, p. 529.

4igoroux: Gazette des Hôpiteaux, 1876, p. 788.

5 Dehio: St. Petersburg Medicine, Wochen

White and Sumunications, Mass. Medical Society, Vol. xix, p. 930.

7 Gibson: Diseases of the Heart and Aorta.
8 Romberg: Ebstein Schwalbe Handbuch der Practischen 8 Romberg:
Medicine, p. 801 .

${ }_{9}$ Baginsky: Diptherie und Diptheritischer Croup, p. 121

10 Romberg: Ebstein Schwalbe Handbuch, p. 952

11 Stokes: Diseases of Heart and Aorta, 185

12 Huchard: Traité Clinique des Maladies du Cœur et le d'Aorta, 899.

${ }^{13}$ Luce: Archiv für Klin. Medicine, 1902

14 Von Schuster: Zurcardialer Bradycardie, Deutsch Medicine, Wochenschrift, 1896 .

15 Hoffman: Zur Kenntniss der Adams-Stokes Krankheit.

itschrift fur Klin. Medicine, 1900

${ }^{16}$ Korcziniski: Centralblatt für Klin. Medicine, 1877.

17 Hammer: Wiener Medicine Wochen.

1s Samuelson: Zeitschrift für Klin. Medicine, Vol. 2, 1881.

19 Von Frey.

${ }^{20}$ Neuberger und Edinger: Berlin Klin. Wochen., 1898, 4

${ }^{21}$ Zurhelle: Krankheiten der periphereschen nerven. Nothnagel, xi, I, g. 241.

${ }_{23}^{23}$ Lace: Deutsches Archiv für Klin. Medicine, 1902.

ymptom der Basilar Thrombose.

21 Strübing: Deutsche Med. Woch., 1893. 